\title{
Determinação do teor de nitrito em amostras de salsicha industrializada
}

\author{
Determination of nitrite content in industrialized sausage samples
}

SANDro Rostelato-Ferreira ${ }^{1}$

Rosangela Honorato Dantas ${ }^{1}$

BeAtriz Gulli Bidoia ${ }^{1}$

${ }^{1}$ Universidade Paulista (UNIP), Sorocaba/SP - Brasil.
Resumo Introdução: Produtos industrializados, como a salsicha, vêm para atender às necessidades da população da vida moderna, considerando que se trata de um alimento de preparo rápido e econômico. Por sua vez, refere-se a um alimento industrializado que contém grandes quantidades de agentes conservantes, como o nitrito de sódio. Овјетіvo: Determinar o teor de nitrito em amostras de salsicha comercializadas no município de Sorocaba-SP. MATERIAIS E MÉTODOs: Foram coletados quatro tipos de amostras de salsicha de diferentes marcas comercializadas nacionalmente (A, B, C e D). O método utilizado foi da desproteinização das amostras, seguido de reação colorimétrica e analisadas em espectrofotômetro. Resultados: Os valores obtidos para as amostras foram, respectivamente: A: $5,15 \mathrm{mg} / \mathrm{kg}$; B: $4,25 \mathrm{mg} / \mathrm{kg}$; C: 5,8 mg/kg; D: 2,75 mg/kg. O valor máximo preconizado pelo Ministério da Agricultura é que seja $1,5 \mathrm{mg} / \mathrm{kg}$. Conclusão: Conclui-se que as marcas analisadas estavam acima do limite máximo permitido pela legislação vigente.

Palavras-chave: Nitrito. Produtos cárneos. Toxicologia dos ALIMENTOS.

Abstract Introduction: Processed products such as sausage, comes to meet the needs the people of modern life, considering that it is a food fast and economical preparation. On the other hand, refers to some processed foods containing large quantities of preservatives such as sodium nitrite. ОвJECTIVE: To determine the nitrite content in sausage samples commercialized in the city of Sorocaba-SP. MATERIAL AND METHODS: We collected four types of sausage samples of different brands marketed nationally (A, B, C and D). The methodology used was the deproteinization of samples, followed by colorimetric reaction and analyzed in a spectrophotometer. REsults: The values obtained for the samples were: A: $5.15 \mathrm{mg} / \mathrm{kg}$; B: $4.25 \mathrm{mg} / \mathrm{kg}$; C: $5.8 \mathrm{mg} / \mathrm{kg}$; D: $2.75 \mathrm{mg} /$ $\mathrm{kg}$. The maximum recommended by the Ministry of Agriculture is to be $1.5 \mathrm{mg} / \mathrm{kg}$. Conclusion: We conclude that the analysed brands were above the maximum extent permitted by law.

Key-words: Nitrite. Meat products. Food toxicology. 


\section{INTRODUÇÃO}

A globalização e o avanço tecnológico fizeram que a população mundial passasse a consumir cada vez mais alimentos que sejam de preparo facilitado e que não tragam riscos à saúde. ${ }^{1}$

A rotina da vida moderna, aliada à falta de tempo para o preparo de alimentos, têm conduzido as indústrias alimentícias a desenvolver produtos de fácil preparo. Nesse contexto, o desenvolvimento de produtos industrializados, como a salsicha, alimento embutido muito consumido pelos brasileiros, ${ }^{2}$ vem para atender a essas necessidades, considerando que se trata de um alimento de preparo rápido, além do baixo custo. ${ }^{3}$

Alimentos industrializados apresentam maior durabilidade, devido à presença de aditivos alimentares com finalidade de conservação desse produto, como é o caso do nitrito de sódio que é adicionado em produtos cárneos para conferir a cor vermelha e sabor característico, além de prevenir alterações desagradáveis e atuar como conservante, principalmente contra o crescimento e a produção de toxina do Clostridium botulinum. ${ }^{4,5,6}$

O maior problema associado à ingestão do nitrito de sódio está relacionado com o seu potencial efeito tóxico em indivíduos expostos, o que depende tanto da quantidade ingerida quanto da susceptibilidade do organismo. $\mathrm{O}$ excesso desses íons causa danos à saúde humana, e é preocupante sob o ponto de vista toxicológico, uma vez que o nitrito é convertido em $\mathrm{N}$-nitrosaminas, e possui ação carcinogênica após a digestão. ${ }^{7}$

Desse modo, o objetivo do presente trabalho foi determinar o teor de nitrito em di- ferentes marcas de salsicha que são conhecidas nacionalmente e comparar com o valor preconizado pela legislação vigente.

\section{MATERIAIS E MÉTODOS}

\section{Amostras}

A pesquisa fundamentou-se em verificar a presença de nitrito em amostras de salsicha de marcas comercialmente conhecidas, que foram identificadas com as letras A, B, C e D.

Foram adquiridas quatro marcas comerciais distintas de salsicha no município de Sorocaba-SP. Após a coleta, foram submetidas à refrigeração até o momento da análise. As análises ocorreram no período entre os meses de março e abril de 2016, e foram realizadas em duplicatas.

O método utilizado foi descrito pela AOAC (Official Methods of Analysis of the Association of Official Analytical Chemists), por análise de espectrofotometria.

As amostras foram pesadas ( $10 \mathrm{~g}$ cada amostra) e trituradas, em seguida, foram realizadas as etapas de desproteinização das amostras utilizando uma solução de bórax e a de clarificação feita por meio do ferrocianeto de potássio e acetato de zinco, de acordo com Teixeira et al. ${ }^{8}$ Após essa etapa, a solução foi filtrada e mantida em banho-maria, a $30^{\circ} \mathrm{C}$ por $30 \mathrm{~min}$, para que a reação de diazotação ocorresse. $\mathrm{O}$ nitrito foi determinado espectrofotometricamente a $474 \mathrm{~nm}$ após a reação de diazotação. Para a reação de diazoconjunção, na formação de cromógeno, foi utilizado o reativo alfa naftol com solução tampão de pH 9,6 - 9,7.

Foi realizada, previamente, uma curva de calibração a partir de uma solução pa- 
drão de nitrito. Para determinar o teor residual de nitrito nas amostras, foi utilizada a equação para a curva de calibração e os resultados estão apresentados como média dos valores obtidos.

Equação:

$C d=A d=F c \rightarrow C d=A d x 14$

Onde:

Cd Concentração do desconhecido

Fc Média de concentração padrão/Absorbância padrão

Ad Absorbância desconhecida

Para determinar os valores de nitrito nas amostras de salsicha, foram, previamente, obtidos os valores de absorbância de concentrações conhecidas de nitrito, conforme apresentados na Tabela 1 .
Tabela 1: Valores médios de nitritos e suas respectivas absorbâncias

\begin{tabular}{cc}
\hline Nitrito $(\mathbf{m g})$ & Absorbância $(\mathbf{n m})$ \\
\hline 0,5 & 0,028 \\
\hline 1 & 0,05 \\
2 & 0,172 \\
4 & 0,215 \\
8 & 0,642 \\
\hline
\end{tabular}

A partir desses dados, foi possível obter a Curva de Calibração (Fig. 1), e cada ponto corresponde à média dos valores obtidos na análise das duplicatas. Os dados apresentados evidenciam linearidade satisfatória no intervalo selecionado, com coeficiente de determinação de $\mathrm{R}^{2}=0,9586$, a qual oferece confiabilidade nos dados da pesquisa.

Figura 1: Curva Padrão da Solução de nitrito de sódio.

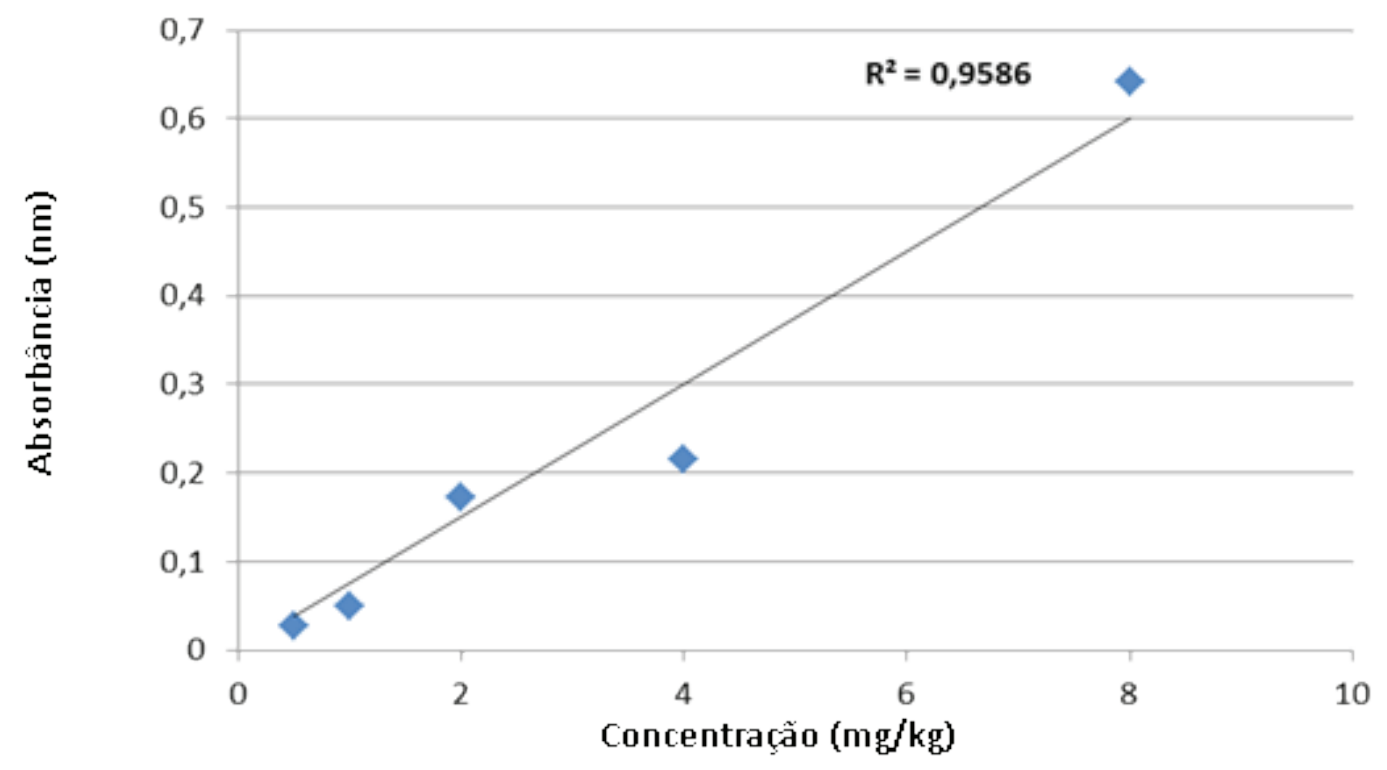


Os dados obtidos para a determinação de nitrito foram tratados estatisticamente por meio do método de Teste $t$, para amostras independentes, usando o programa OriginPro8.

\section{RESUltados}

Os resultados de nitrito residual foram obtidos a partir da solução desproteinizada das amostras de salsicha, e estão apresentados na Tabela 2. Os resultados demonstram que todas as marcas analisadas ultrapassaram o limite máximo permitido.

Tabela 2: Concentração de nitrito de sódio em salsicha de marcas comerciais distintas

\begin{tabular}{lll}
\hline Amostras & \multicolumn{1}{c}{$\begin{array}{c}\text { Concentração } \\
(\mathbf{m g} / \mathbf{k g})\end{array}$} & $\begin{array}{c}\text { Referência } \\
(\mathbf{m g} / \mathbf{k g})\end{array}$ \\
\hline $\mathrm{A}$ & $5,15^{\star}$ & \\
$\mathrm{B}$ & $4,25^{\star}$ & $1,5 \mathrm{mg} / \mathrm{kg}$ \\
$\mathrm{C}$ & $5,8^{\star}$ & \\
$\mathrm{D}$ & $2,75^{\star}$ & \\
\hline
\end{tabular}

${ }^{\star}$ Diferença significativa quando comparada à referência

As diferentes marcas comerciais de salsicha estavam acima do limite permitido pela legislação vigente, de acordo com a técnica utilizada para a determinação de nitrito em produtos cárneos.

\section{Discussão}

No Brasil, o limite máximo permitido de nitrito utilizado como conservante até 1998 era de $200 \mathrm{mg} / \mathrm{kg}$, sendo reduzido a partir dessa data para $150 \mathrm{mg} / \mathrm{kg}$, por meio da por- taria $n^{\circ} .1 .004$, de 11 de dezembro de 1998 . Em 2006, houve alteração nessa portaria a qual estabeleceu um novo valor para o nitrito residual de 0,015g/100g ou 1,5mg/kg. Assim, em proteção à Saúde Pública, os níveis de nitrito têm sido reduzidos em carnes curadas, objetivando-se a diminuição da formação de nitrosaminas, entre outros compostos. ${ }^{9,10}$

Os resultados aqui observados corroboram com os achados de Ferreira Freire et al., ${ }^{11}$ que verificaram a presença de nitrito em salsichas comercializadas na cidade de Lavras-MG, observando que todas as amostras analisadas ultrapassaram o limite de nitrito que é permitido.

Estudo análogo por Melo Filho et al. ${ }^{12}$ avaliou a qualidade de salsichas comercializadas no município de Recife-PE. Os níveis de nitrito residual foram determinados em 54 amostras de salsichas tipo hot dog, e todas as amostras apresentaram níveis de nitrito acima do limite máximo.

Souza Guerreiro et al. ${ }^{13}$ avaliaram 18 amostras aleatórias de três produtos cárneos adquiridas em supermercado na cidade de Salvador-BA. Dessas amostras, seis foram de mortadela, seis de presunto e seis de salsichas de diferentes marcas comerciais cadastradas no Serviço de Inspeção Federal (SIF). Para as amostras de salsichas analisadas, apenas uma marca não estava em conformidade com a legislação brasileira. As demais amostras apresentaram teores inferiores aos limites máximos permitidos estabelecidos pela legislação brasileira, o que assegura o atendimento às recomendações legais.

Justifica-se que o uso de nitrito como aditivo em alimentos, além de conferir-lhes aspectos de cor e sabor, possui ação preventiva na germinação e proliferação de esporos 
de algumas bactérias, especialmente Clostridium botulinum, responsável pela produção de neurotoxinas causadoras do quadro de botulismo, ${ }^{14}$ embora Rui et al. ${ }^{15}$ afirmem que a temperatura de processamento desses alimentos é mais eficiente que o nitrito no combate ao Clostridium botulinum. Dessa forma, devido às suas características, nenhum aditivo reúne tantas funções de preservação como o nitrito. ${ }^{16}$

Por sua vez, a quantidade exacerbada desses agentes em alimentos pode desencadear efeitos tóxicos, já que a concentração de nitrito, quando alta, é influenciadora na formação de metemoglobina, gerando a metemoglobinemia, podendo provocar cianose, anóxia e morte. A metemoglobina é capaz de oxidar o $\mathrm{Fe}^{2+}$ da hemoglobina, convertendo-o em metemoglobina $\left(\mathrm{Fe}^{3+}\right)$, diminuindo o transporte de oxigênio e sendo capaz de provocar os efeitos já citados. Além do mais, é capaz de influenciar a formação de compostos $\mathrm{N}$-nitrosos, que são fatores estimulantes para o surgimento do câncer, especialmente câncer gástrico, hepático e de esôfago. ${ }^{7}$
Medidas de controle de produção poderiam ser aplicadas com mais rigor em indústrias e estabelecimentos comerciais, com o objetivo de se garantir produtos de maior qualidade e seguros quanto ao teor de nitrito em sua composição. ${ }^{17}$ Ancorado nesse ponto de vista, Cartaxo ${ }^{18}$ sugere que produtos com adição de nitrito só sejam disponibilizados para a venda mediante laudo analítico.

Sendo assim, nota-se que mesmo havendo uma legislação vigente para os cuidados relacionados à quantidade de substâncias adicionadas aos alimentos, é possível encontrar valores acima do limite máximo permitido.

\section{CONSIDERAÇõeS FINAIS}

De acordo com os resultados obtidos em diferentes amostras comerciais de salsicha, pode-se concluir que as marcas identificadas como A, B, C e D apresentaram-se acima do limite máximo permitido pela legislação vigente.

\section{REFERÊNCIAS}

1. Almudema A, Lizaso J. Nitratos, nitritos y nitrosaminas. Madrid: Fundacion Ibérica para La Seguridad Alimentaria, 2001. Disponível em: www.proyectopandora.es/wp-content/uploads/Bibliografia/13181019_nitritos_nitratos.pdf. Acesso em: 15/05/2016.

2. Ferraccioli VR. Avaliação da qualidade de Salsichas do tipo Hot Dog durante o armazenamento. Dissertação [Mestrado em Engenharia de Processos Químicos e Bioquímicos]. Instituto Mauá de Tecnologia. São Caetano do Sul, 2012, p. 116.

3. Petenuci ME, Matsushita M., Souza NE, Visetainer JV. Nitratos e nitritos na conservação de carnes. Rev Nac Carne 2004, v. 333, p. 1-2.

4. Dutra MP, Ramos EM, Ramos ALS, Fontes PR, Cardoso GP, Leal AS. Radiação Gama e Tempo de Armazenamento sobre Oxidação lipídica, cor objetivo, Pigmento Heme e Nitrato Residual de Mortadelas elaboradas com Diferentes Níveis de Nitrato. Ciência Rural 2011, v. 41, n. 12, p. 2.203-9.

5. Nitrini SMOO, Toledo FCP, Berto JF, Rodrigues APO, Nogueira HR, Monti EJ. Determinação de nitritos e nitratos e linguiças comercializadas na região de Bragança Paulista. LECTA-Usf 2000. Bragança Paulista, v. 18, n. 1, p. 91-6. 
6. Lira GM, Silva Neta ML, Souza JB, Barros ES. Teores de nitrito de sódio em produtos cárneos comercializados em Maceió-AL. Rev. Inst. Adolfo Lutz 2003. São Paulo, v. 63, n. 3, p. 165-70.

7. Gonçalves JF, Oliveira WC, Silva CAO, Cunha MRR, Pereira FR. Ocorrência de Nitratos e Nitritos em Queijos Minas Frescal, Mussarela, Parmesão e Prato. Rev Inst Adolfo Lutz 201 1, 70(2): p. 193-8.

8. Teixeira AL, Nascimento AP, Souza DFF, Maia PP. Avaliação dos níveis de Nitrato e Nitrito em amostras de Bebidas Lácteas comercializadas na cidade de Lavras/MG. Rev Univ Vale do Rio Verde. Três Corações, v. 12, n. 1, p. 85-9.

9. Brasil. Ministério da Saúde. Agência Nacional de Vigilância Sanitária. Portaria no ${ }^{\circ}$ 540. Brasília, DF, 27 out. 1997. Aprova o Regulamento Técnico: Aditivos alimentares.

10. Martins DI, Midio AF. Toxicologia dos alimentos, 2 ed. São Paulo: Varela; 2000, 295p.

11. Ferreira Freire HM, Moreira EA, Freitas DF. Avaliação dos níveis nitrato e nitrito em salsichas comercializadas na cidade de Lavras/MG. Rev Univ Vale do Rio Verde. Três Corações, 2014, v. 11, n. 2, p. 218-27.

12. Melo Filho AB, Biscontini TMB, Andrade SAC. Níveis de nitrito e nitrato em salsichas comercializadas na região Metropolitana do Recife. Ciênc Tecnol Alimentos. Campinas 2004, 24(3): p. 390-92.

13. Guerreiro RS, Sá MS, Rodrigues LAP. Avaliação do teor de nitrito e nitrato em alimentos cárneos comercializados em Salvador. RevInter Revista Intertox de Toxicologia, Risco Ambiental e Sociedade 2012, v. 5, n. 1, p. 77-91.

14. Ordoñez J., Rodriguez A., Àlvarez L., Sanz M., Minguillong G., Perales L., et al. Tecnologia de Alimentos: Alimentos de origem animal, v. 2. Porto Alegre: Artmed, p. 280, 2005.

15. Hui YH, Wai-Kit N., Rogers R. Meat Curing Technolog: Meat Science and Applications. New York: Marcel Dekker, p. 704, 2001.

16. Perigo JA, Whiting E., Bashford TE. Observations on the inhibition of vegetative cells of Clostridium sporogenes by nitrite which has been autoclaved in a laboratory medium, discussed in the context of sub-lethally processed cured meats. Internat Journ Food Scien Technol 1967, 2: p. 377-97.

17. Oliveira JF, Silva UR, Pastore VAA, Azevedo EC, Campos GM, Silva FCG, et al. Determinação espectrofotométrica de nitrito em produtos cárneos embutidos. Revista Brasileira de Higiene e Sanidade Animal 2017, 11:1, p. 19-31.

18. Cartaxo JLS. Riscos associados aos níveis de nitritos em alimentos: uma revisão. 30p. Trabalho de Conclusão de Curso, Universidade Federal da Paraíba. João Pessoa-PB, 2015.

\section{Dados dos Autores}

\section{SANDro Rostelato-Ferreira}

Doutor em Farmacologia pela Universidade Estadual de Campinas. Docente nos Cursos de Farmácia e Biomedicina na Universidade Paulista. Sorocaba/SP - Brasil. sandrorostelato@yahoo.com.br

\section{Rosangela Honorato Dantas}

Graduada em Biomedicina pela Universidade Paulista. Sorocaba/SP - Brasil. honorato.rosangela@ hotmail.com

\section{Beatriz Gulli Bidoia}

Doutora em Ciências (Hematologia) pela Universidade Federal de São Paulo. Docente no Curso de Biomedicina na Universidade Paulista, Sorocaba/SP - Brasil. beatriz_bidoia@hotmail.com

Submetido em: 10-8-2016

Aceito em: 5-6-2017 